\title{
Hydroxyapatie Formation and Its Interaction with Osteoblastic Cells
}

\author{
H. Vali, ${ }^{*}$ P. Ghiabi, ${ }^{*}$ J. Henderson, $^{* *}$ M.D. McKee, ${ }^{* * *}$ E. Chevet, ${ }^{*}$ and S.K. Sears* ${ }^{1}$
}

*Electron Microscopy Centre, Department of Anatomy \& Cell Biology, McGill University,3640 University Street, Montreal, QC H3A 2B2

** Lady Davis Institute, Jewish General Hospital, Montreal, Quebec H3T 1E2

*** Faculty of Dentistry/Department of Anatomy \& Cell Biology, McGill University, Montreal, QC H3A 2B2

Uncertainty remains over the interpretation of nano-phenomena from various biological and geological environments as living organisms. A characteristic feature of nano-phenomena is their association with a variety of mineral phases. One of the most controversial issues involving nanophenomena is the existence of Nanobacterium sanguineum isolated from human and cow sera [1]. A unique trait associated with this nano-organism is the formation of hydroxyapatite (HA). The nature and formation of the HA phase(s), however, is still not well understood in this system. It is clear that HA is associated with many pathological calcifications, including kidney stones, atherosclerosis, urolithiasis, calcification in hemodialysis patients, dental plaque, and dysfunctional calcification occurring in implanted cardiovascular devices. Although our results do not confirm bacteria controlled or induced mineralization leading to formation of thesemineralized nanoforms, the association of HA with serum protein suggests a biogenic origin.[2] To investigate the interaction of nanoforms with MC3T3-E1 cells and the mechanism(s) of calcification, several sets of experiments were conducted under variable cell culture conditions. Extensive variation in morphology occurs between HA in nanoforms obtained from 10\% FBS/DMEM (Fig 1a) and HA present in MC3T3-E1 cell cultures (Figs. 1b-f). Aggregates of altered nanoforms were present within a membranous organelle (Figure 1c, d). Occasionally, vacuole-like structures containing isolated crystals were observed that are likely a result of the disintegration of nanoforms. The original structure of nanoforms was not preserved either outside or inside of the cells. The distribution and concentration of mineralized structures within the cells differed extensively depending on the composition of the growth medium. The mineralized structures in the experiments conducted with nanoforms grown in EMEM show high concentrations of HA crystals within the cells. Larger well-ordered crystals, showing evidence of newly formed HA, are observed within membrane-bound regions (Fig. 1f). It is likely that the changes in size and morphology of the HA crystals are the result of the dissolution of the fine-grained HA crystals associated with the nanoforms obtained from serum (Fig. 1a) and the neoformation of distinct HA crystals observed in MC3T3-E1 cells (Fig. 1f). The mechanism of transformation and the involvement of MC3T3-E1 cells in this reaction are not understood. It may be that within the membrane-bound phagocytic compartments observed in the MC3T3-E1 cells, different regulatory controls are exerted over crystal growth that otherwise would occur extracellularly, thus resulting in the formation of large crystals. Further study mayreveal the significance of these experiments for pathological calcification and bone formation. However, the approach used in this study provides a unique opportunity to understand the mechanisms of HA formation in both normal and pathological calcification. In addition, this process may eventually be used for bone tissue engineering where mineralization is desirable with synthetic bone substitutes.

[1] E.O. Kajander \& N. Ciftcioglu Proc. Natl. Acad. Sci. USA 95 (1998) 8279.

[2] H. Vali et al., Geochim. Cosmochim. Acta 65 (2001) 63. 

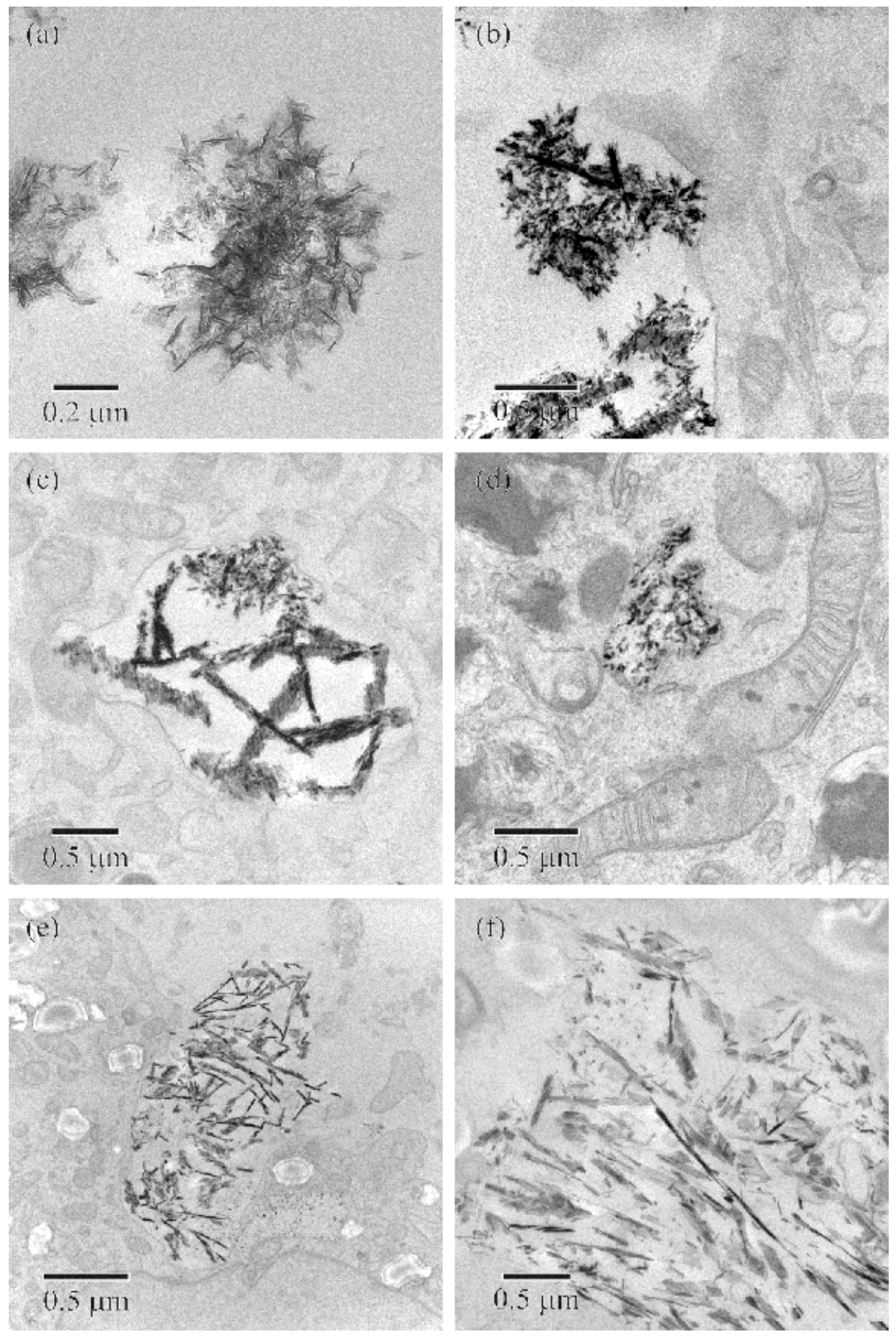

FIG. 1. TEM images of ultrathin sections obtained from nanoforms: (a) nanoforms obtained from 10\% FBS/DMEM; (b) nanoforms exposed to MC3T3-E1 cells showing the stage of internalization; (c) internalized aggregates of HA showing a unique distribution; (d) dispersed HA crystals with dissolution features; (e) a large aggregate of HA engulfed by the cell; (f) large neoformed HA crystals present within a MC3T3-E1 cell. 